\title{
Double Cortex Syndrome
}

National Cancer Institute

\section{Source}

National Cancer Institute. Double Cortex Syndrome. NCI Thesaurus. Code C116933.

A developmental brain abnormality characterized by atypical migration of neurons during cortical development. 